\title{
P2P Layered Video Streaming over Wireless Ad Hoc Networks
}

\author{
N. N. Qadri \\ COMSATS Institute of Technology \\ Islambad \\ Pakistan \\ +92 (051) 9258481-5 \\ nadianqadri@googlemail.com
}

\author{
M. Fleury \\ University of Essex \\ Colchester CO4 3SQ \\ United Kingdom \\ +44 1206-872434 \\ fleum@essex.ac.uk
}

\author{
M. Altaf \& M. Ghanbari \\ University of Essex \\ Colchester CO4 3SQ \\ United Kingdom \\ +44 1206-872434
}

\{maltaf, ghan\}@essex.ac.uk

\begin{abstract}
In this paper, we consider multicast video clip streaming in an ad hoc network. Efficient delivery of video is accomplished by means of a Peer-to-Peer (P2P) overlay network. To exploit multipath transport the paper introduces layered video streaming without the need for base layer protection. In an urban environment with higher node densities and lower node speeds, the network performance is shown to be stable and superior compared to single-layer distribution. Detailed analysis confirms the results predicted by summary statistics. Heterogeneous wireless networks are targeted in which ad hoc networks relieve cellular wireless networks.
\end{abstract}

\section{Categories and Subject Descriptors}

C.2.1 [Computer-Communication Networks]: Network Architecture and Design-Wireless communication

\section{General Terms}

Algorithms, Experimentation, Performance.

\section{Keywords}

Ad hoc network, layered video, multi-path transport, P2P.

\section{INTRODUCTION}

Wireless ad hoc networks can: relieve congested cells, extend coverage, and service dead-spots within cellular networks. This is accomplished by dual cellular and ad hoc interfaces on mobile phones [1] or by relay stations [2] for those mobile phones not so equipped. In the 3GPP, the Multimedia Broadcast/Multicast Service (MBMS) [3] has been developed to distribute video over cellular networks and it is natural to ask if an ad hoc network could extend such a service. In this paper, it is assumed that video clips, originating in a cellular network, are distributed to a group of nodes by means of an ad hoc network. Though the paper

Permission to make digital or hard copies of all or part of this work for personal or classroom use is granted without fee provided that copies are not made or distributed for profit or commercial advantage and that copies bear this notice and the full citation on the first page. To copy otherwise, or republish, to post on servers or to redistribute to lists, requires prior specific permission and/or a fee.

MobiMedia'09, September 7-9, 2009, London, UK.

Copyright 2009 ICST 978-963-9799-62-2/00/0004 ... \$5.00 investigates scenarios in which the nodes (mobile phone carriers) move at differing speeds and the node density is varied, in the urban settings in which congested cells are likely to occur, node densities could be high and average node speeds could be slow as many carriers could be on foot.

An interesting way to achieve distribution over an ad hoc network is through Peer-to-Peer (P2P) application-oriented overlays [4]. A good number of decentralized P2P streaming systems have been deployed to provide live and on-demand streaming services on the fixed Internet and the same ideas may be useful in providing realtime streaming in ad hoc networks. Despite being different paradigms, wireless ad hoc networks and P2P both benefit from multi-source distribution and multi-path delivery [5].Therefore, in this paper video is distributed across a P2P overlay network.

Multi-path video distribution is also attractive for ad hoc networks because if packet loss, delay or jitter occurs on one of the paths then this can be compensated for by the encoded bit-stream arriving over other paths. In fact, ad hoc networks are also prone to link breakages because of the motion of the carriers. Multi-path streaming may reduce the bandwidth requirement [6] for any one route through an ad hoc network, at a possible cost in increased coding redundancy. Multiple Description Coding (MDC) is a multi-path streaming solution with a role in wireless [7] and $\mathrm{P} 2 \mathrm{P}$ streaming [8]. At times when two or more MDC streams arrive at a destination, they provide an enhanced quality video stream, whereas if just one stream arrives it is still watchable but is coarser than the MDC version. Unfortunately, MDC [9] requires specialist codecs and is computationally complex, because in general it requires synchronization between encoder and decoder to reduce motion estimation error drift. Simplified versions of MDC are possible [10] but in this paper we consider layered coding.

In classical layered coding, a base layer and one or more enhancement layers provide scalable video quality [11]. However, unlike MDC the base layer should be successfully delivered as the video cannot be reconstructed without it. In earlier experiments with layered video $[12,13]$, if base layer packets were lost, this problem had to be resolved by means of an Automatic Repeat ReQuest (ARQ) message. As latency in an ad hoc network is variable and can be high, there were a limited number of settings in which layered video was competitive with MDC.

However, in the low bitrate H.264/Scalable Video Codec (SVC) one option is to output one stream consisting of base layer and 
enhanced layer, and another stream consisting of the base layer alone. In this variety of layered video, if packets from the layered stream fail to arrive, the other stream may still serve to reconstruct the video, whereas if packets from the combined base and enhanced layer stream are lost base layer packets may still be available. This idea appears to be self-defeating because of the extra bandwidth consumed by the combined stream.

However, in an ad hoc network if this bandwidth is not consumed then it is not used, while in contrast in a cellular network all communication is over one hop to the master control station. Therefore, a further contribution of this paper, apart from the introduction of mesh-P2P streaming over ad hoc, is to show that this variety of layered coding not only does not consume greater overhead or increase delay compared to single stream transport of the same stream but on average it also improves network delivery, especially within an urban environment. It is important to emphasize that this form of layered coding does not require protection of the base layer stream, as effectively the base layer is sent over both paths.

Because the P2P system tested involves multicast distribution of video the network performance of the delivery system will vary between destination nodes. Simulations show that the average (arithmetic mean) performance of layered video distribution is superior to single stream but that for any one distribution there will be some destination nodes that do not benefit compared to single stream distribution. In fact, depending on speed and node density within the ad hoc network, it will certainly be necessary for some destination nodes to request redistribution of the clip (or some other remedial action), possibly over the cellular network rather than the ad hoc. However, the average network performance of layered coding was found to be superior in the simulations.

The remainder of this paper is organised as follows. Section 2 reviews previous work around multi-path video streaming, while Section 3 introduces P2P overlay upon ad hoc networks. Section 4 details the simulation methodology. Section 5 presents our results, with Section 6 finally drawing some conclusions.

\section{RELATED WORK}

\subsection{Multi-path video over ad hoc networks}

Early research in [14] examined point-to-point Constant Bit Rate (CBR) video streaming in the 15-node network tested over a 1000 $\times 1000 \mathrm{~m}^{2}$ area. The source video was simply partitioned into multiple streams and paths were selected according to congestion conditions. The reference 'Foreman' Quarter Common Intermediate Format (QCIF) video clip at $30 \mathrm{~Hz}$ (frame/s) was simulated at rates ranging from around $50 \mathrm{kbps}$ up to $350 \mathrm{kbps}$. Group of Pictures (GOP) sizes were varied, with playout buffer settings equivalent to $350 \mathrm{~ms}$ and $500 \mathrm{~ms}$ of video. The paper reported that as the number of multi-path routes increased to six, the delivered video quality increased. Unfortunately, the work in [14] did not report on node mobility or radio range. However, the paper did show that optimal regimes exist but that simple formulas require perfect network traffic knowledge by each node, which is impractical.

In [15] rather than simple partitioning of the video for a more detailed examination, MDC was employed. A denser node distribution (60 nodes in a $1200 \times 800 \mathrm{~m}^{2}$ area) was chosen in [15] with the well-known random waypoint [16] mobility model and with maximum speeds varying from $2.5 \mathrm{~m} / \mathrm{s}$ to $15 \mathrm{~m} / \mathrm{s}$. The playout buffer size was $100 \mathrm{~ms}$ of video storage with video streamed at a rate of $192 \mathrm{kbps}$ for $12 \mathrm{~Hz}$. Radio range was $250 \mathrm{~m}$ for an IEEE 802.11 wireless LAN in ad hoc mode but the node pause period was not given in the paper. Because of the node density, the effects of mobility were not strongly felt, as it is not possible for nodes to quickly lose radio contact with surrounding nodes (resulting in broken wireless links). Though Reed-Solomon (RS) FEC was employed in simulations, it should be borne in mind that this form of FEC has quadratic computational complexity, which may overwhelm battery-powered devices. The paper showed the advantage of the authors' multiple tree algorithm for video multicast.

A paper by the same authors [17] amongst other results showed that, provided the paths were disjoint, IEEE 802.11's Carrier Sense and Medium Access with Collision Avoidance (CSMA/CA) is unlikely to lead to traffic interference. This line of research was continued in the authors' most recent contribution at the time of writing [18], in which the robustness of the paths is estimated in advance. For example, the received signal strength could be reported along with the level of contending cross-traffic. The work's strength is that physical tests have now confirmed the findings.

\subsection{Distribution of video over multi-paths}

A number of alternative ways of taking advantage of path diversity have been investigated. In [12], transfer of a base layer and one or more enhancement layers over multi-paths was combined with ARQ. In this form of layered video if the base layer is not received correctly, the decoder cannot reconstruct the original video. By assuming that the display deadline was twice the round-trip time $(300 \mathrm{~ms})$, it is possible to send one ARQ to protect the base layer. A realistic channel model with 'bursty' errors and path breakdowns was assumed. The ARQ scheme was shown to improve Peak Signal-o-Noise Ratio (PSNR) by up to 10 $\mathrm{dB}$, upon sending the layered video over multi-paths without ARQ.

In a general context, the research in [19] concluded that layered video is competitive if the rate is modified according to the distortion. Rate-distortion analysis is now a built-in facility of the H.264 codec and can be simplified to reduce the computational overhead. In [13], two further multi-path schemes were compared with layering combined with ARQ, namely: 1) feedback requesting reference frames; and 2) a variant of MDC with motion compensation. In the first of these approaches, the problem of decoder-encoder synchronization was tackled by sending a negative ARQ to indicate the most recent successfully received reference frame upon which motion compensation can be based. Therefore, this scheme also assumed sufficient playout time and bandwidth to allow ACKs. Sending ACKs will also cause more control packet overhead, which can be high. In the variant of MDC tested, no ARQs were sent but a correction method at the decoder was necessary to counter drift between decoder and encoder. The CSMA/CA MAC was assumed with a multipath variant of the reactive Dynamic Source Routing (DSR). The authors concluded that acceptable video quality is possible but which scheme is selected is dependent on the ad hoc scenario. If this uncertainty is to be avoided, then layered video should be more robust, which is one intention of our paper. 
To overcome the problem of FEC complexity, rateless coding with linear decoder complexity was employed in [20] to protect the video layers. Unequal layer protection allows the receiver to control the number of layers and protection level it receives. Though the scheme is formally described as multi-source, it is close to P2P distribution. Unfortunately, the extent of physical layer modeling is unclear and, therefore, so is the effectiveness.

\section{P2P OVER AD HOC}

We have employed a P2P overlay upon an ad hoc network. Both ad hoc and P2P networks are decentralized, autonomous and highly dynamic in a fairly similar way. In both cases, network nodes contribute to the overall system performance in an intermittent and unpredictably manner but nonetheless exhibit a high level of resilience and availability. Fig. 1 illustrates a P2P application overlay over an ad hoc network, in which an overlay network is placed over the network layer. It is important to note that the overlay node placement is logically different to that of the physical placement of the nodes.

The various approaches to P2P streaming have been surveyed by Liu et al [21]. Two main topologies have emerged, i.e. tree-based and mesh-based P2P. Mesh-P2P streaming is flexible and can be managed easily in comparison to a tree-based topology. Moreover, it is not affected by the churn of peers or the effects of handover. A mesh-based topology can also overcome the bandwidth heterogeneity present in an ad hoc network. Consequently, we believe mesh-P2P could be an effective solution for an ad hoc network.

The mesh-P2P overlay tested is shown in Fig. 2. We have used seven nodes to form a mesh out of which two nodes are source nodes. Three nodes (peer C, peer D and peer E) join with these source nodes to retrieve the video content. These three nodes then connect to two further nodes (peer $F$ and peer $G$ ) to serve the video contents that they are to receive from peers $\mathrm{A}$ and $\mathrm{B}$. Here, nodes C, D and E download and upload at the same time. The packetized data streams in Fig. 2 represent layered video streams sent over different paths for protection against channel error However, the packet scheduling algorithm and peer selection or

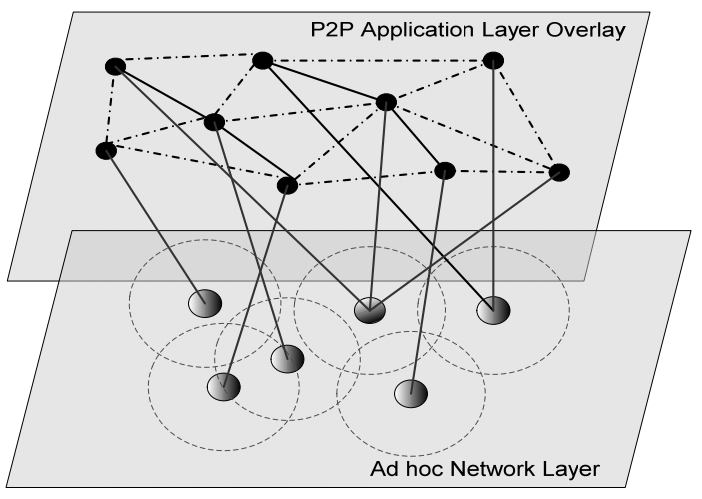

Ad hoc Node

P2P Node

Connection b/w P2P nodes

Connection b/w P2P node and Ad hoc node

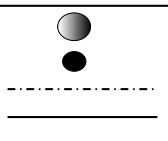

Figure 1. An example of a P2P application overlay over an ad hoc network, after [4].

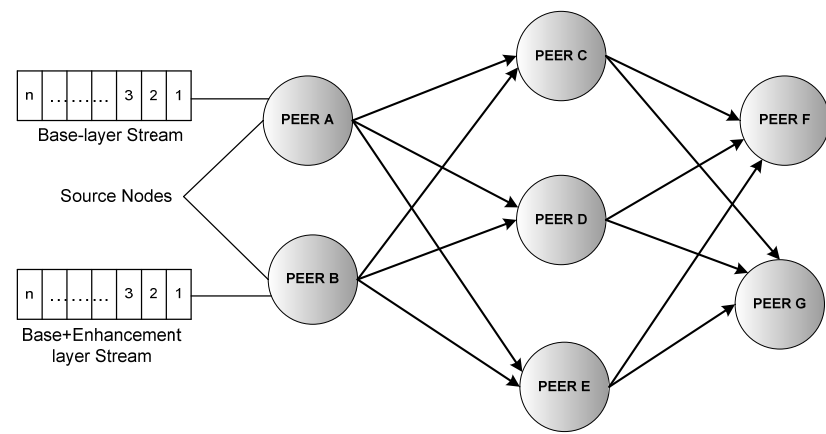

Figure 2. Mesh-based P2P topology sending data streams from sources to receivers.

querying mechanism is beyond the scope of our paper and we assume that it has been achieved.

\section{SIMULATION ENVIRONMENT}

The Global Mobile System Simulator (GloMoSim) [22] simulation library was employed to generate our results. GloMoSim was developed based on a layered approach similar to the OSI seven-layer network architecture. IP framing was employed with UDP transport, as TCP transport can introduce unbounded delay, which is not suitable for delay-intolerant video streaming. Simulations results were averaged (arithmetic mean) over 50 runs, with $95 \%$ confidence intervals established for each data point.

The Ad-hoc On demand Distance Vector (AODV) reactive routing protocol [23] was selected as it does not transmit periodic routing messages, which, for proactive, table-driven protocols, can result in greater control overhead unless network traffic is high. A disadvantage of a reactive protocol is the latency introduced by the route discovery process, which is judged in these simulations for its impact on video end-to-end delay. At the data-link layer, CSMA/CA MAC was set up, as previous studies (refer to Section 2) also mostly assume IEEE 802.11 wireless systems. Therefore, these choices make it easier to interpret the results.

The default parameters and variable ranges for the simulations are summarized in Table 1. GloMoSim provides a two-ray channel model with antenna height hardwired at $1.5 \mathrm{~m}$, and with a Friss free-space model with parameters (exponent, sigma) $=(2.0,0.0)$ for near line-of-sight and plane earth path loss $(4.0,0.0)$ for far line-of-sight, with cross-over distance about $86 \mathrm{~m}$. The radio range was $250 \mathrm{~m}$ with $1 \mathrm{Mbps}$ shared maximum data-rate. Setting the bandwidth capacity to the latter value in the simulation allows modeling of a limited available bandwidth.

The random waypoint mobility model was employed with a default number of 50 nodes in a roaming area of $1000 \times 1000$ $\mathrm{m}^{2}$. In this model, nodes are usually placed randomly in the simulated area. After pausing, the node moves to another random destination at a speed between a minimum and maximum speed. The pause time (time spent once a node reaches its destination) was set to $5 \mathrm{~s}$. The minimum speed was $0 \mathrm{~m} / \mathrm{s}$, while the maximum node speed ranged from 1 to $36 \mathrm{~m} / \mathrm{s}$, i.e. from a slow walk to fast motorbike speeds. However, manual intervention occurred by us in the initial placement of the nodes in such a way that ensured disjoint paths were found by the simulator. After, the initial node placement no further intervention took. 
Table 1. Default parameter settings for the simulations.

\begin{tabular}{|l|l|}
\hline \multicolumn{1}{|c|}{ Parameter } & Value \\
\hline Wireless technology & IEEE $802.11 \mathrm{~b}$ \\
Channel model & Two-ray \\
Max. range & $250 \mathrm{~m}$ \\
Roaming area & $1000 \times 1000 \mathrm{~m}^{2}$ \\
Pause time & $5 \mathrm{~s}$ \\
No. of nodes & $50($ varying $10-100)$ \\
Min. speed & $0 \mathrm{~m} / \mathrm{s}$ \\
Max. speed & $10 \mathrm{~m} / \mathrm{s}$ (varying $1-36 \mathrm{~m} / \mathrm{s})$ \\
Mobility model & Random waypoint \\
Routing protocol & AODV \\
\hline
\end{tabular}

The issue of how to achieve disjoint paths from within AODV is outside the scope of this paper. For a static network, it may be feasible to apply the approach in [19], while in [24] split multipath routing was added to the Dynamic Source Routing (DSR) protocol. The Foreman video clip was Constant Bit-Rate (CBR) encoded in layered form using H264/SVC at Quarter QCIF resolution $(176 \times 144 \mathrm{pixel} /$ frame $)$ with 4:2:0 sampling and Real-Time Protocol file format. Sending at CBR avoids irregular buffering latencies. The frame rate was $15 \mathrm{~Hz}$. In fact, frame rates as low as $10 \mathrm{~Hz}$ [25] may be necessary in an ad hoc network. However, the resulting data rates are not that different from those currently employed for video in $3 \mathrm{G}$ networks, as described in Section 1. Foreman employs the close-up camera shots shown to be suitable for mobile communication [26]. The camera pan towards the end of this sequence increases the coding complexity of the sequence.

\section{RESULTS}

In Fig. 3, the packet loss ratio for single stream and layered video under H.264/SVC are compared. Packet loss ratio is defined by us as the number of packets lost to the number of packets sent. Once the node density is sufficiently high the performance is stable. It is clear that the total packet loss for both streams arriving at any one destination is reduced under SVC compared to sending the equivalent video stream in a single stream. The packet loss ratio is also below $10 \%$ which is the approximate level above which packet loss rates have an effect upon video quality, though somewhat higher levels may be tolerated, as discussed later in this Section. The dispersion of nodes at low densities explains the poor packet loss ratios in these situations, as nodes are unable to come close enough together for sufficient time to easily transfer packets. Fig. 4 presents the packet loss when the node speed varies and the network size is static according to Table 1 . The packet loss appears to increase for single streams relative to layered transport at the slower speeds characteristic of urban environments.

End-to-end delay might be important in the application proposed in Section 1, if the ad hoc network were to be used to divert traffic from a congested cell to another. It also affects playout buffer size at the destination node. End-to-end delay is the time to transport a packet across the multiple hops of its path. At low node densities, Fig. 5, average delay is higher and can be highly erratic, due no doubt to the widely dispersed position of the nodes. Statistically, the two methods of video distribution do not significantly differ at most densities. At slower speeds with medium node densities, Fig. 6 , average delay is reduced. At walking speeds, single stream transport is better but the one way delay would probably be less than $50 \mathrm{~ms}$ whichever method were to be used. Fortunately, these speeds are those envisaged for the target application.

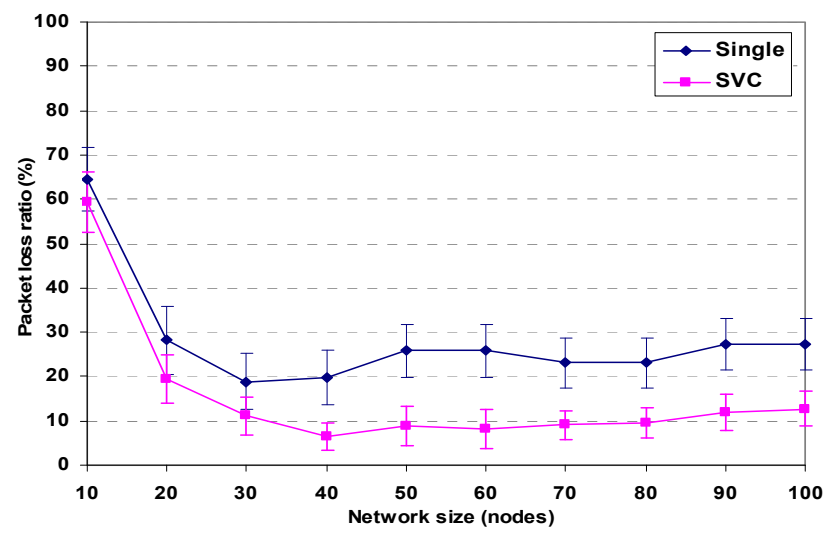

Figure 3. Varying network size with average packet loss ratios for single and layered video transport, showing $95 \%$ confidence intervals.

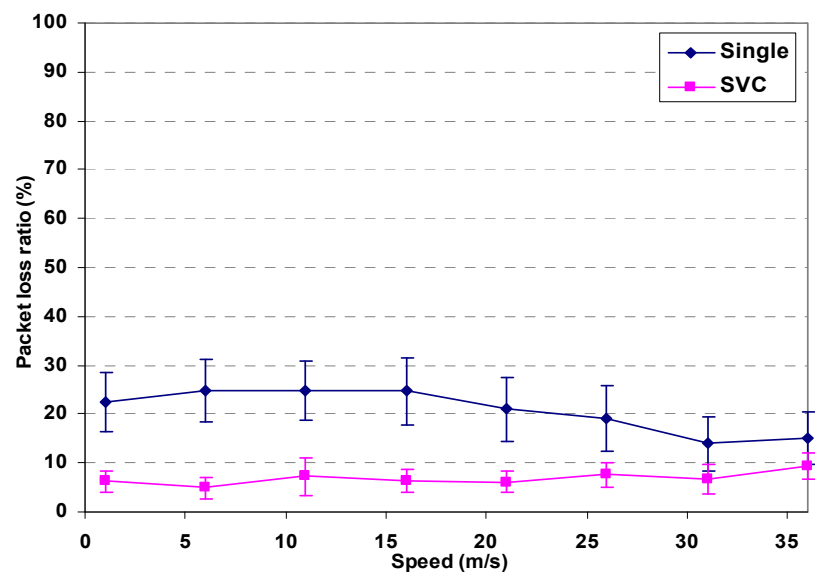

Figure 4. Varying node speed with average packet loss ratios for single and layered video transport, showing $95 \%$ confidence intervals.

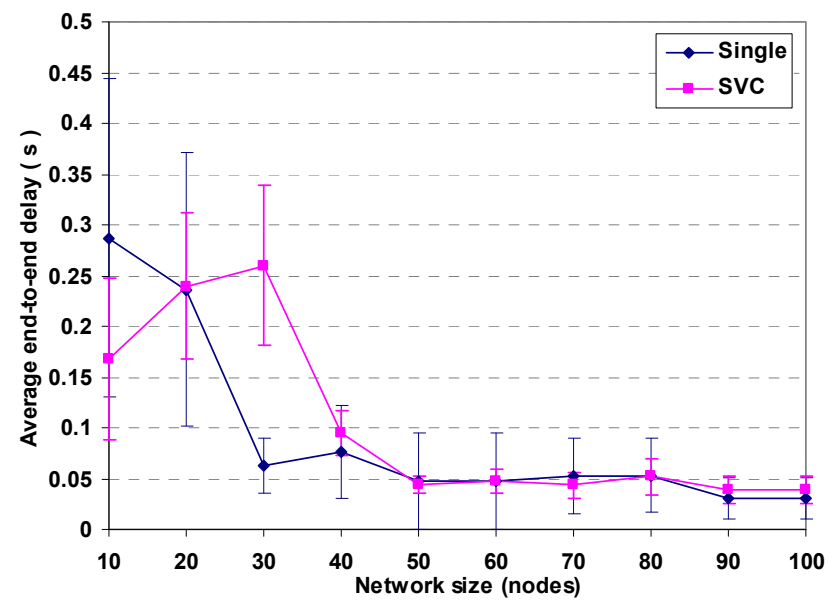

Figure 5. Varying network size with average delay for single and layered video transport, showing $95 \%$ conf. intervals. 


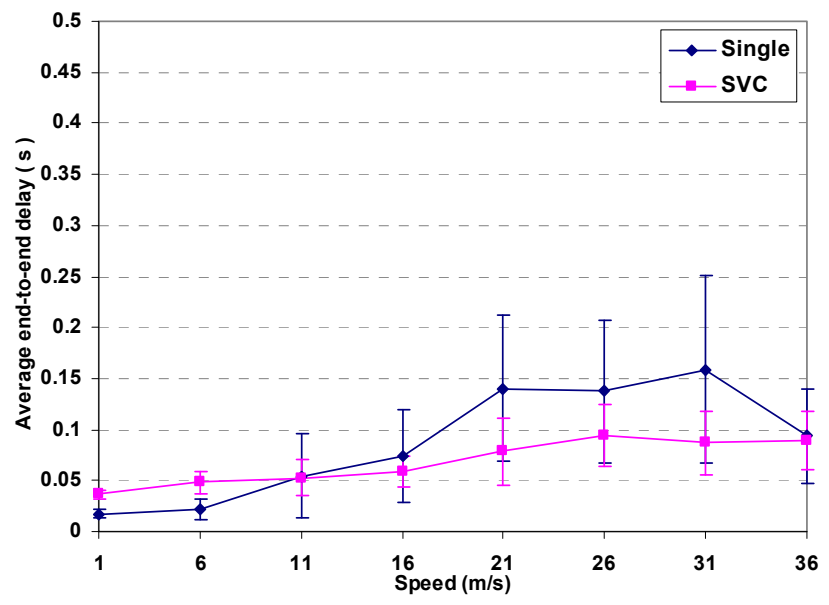

Figure 6. Varying node speed with average delay for single and layered video transport, showing $95 \%$ conf. intervals.

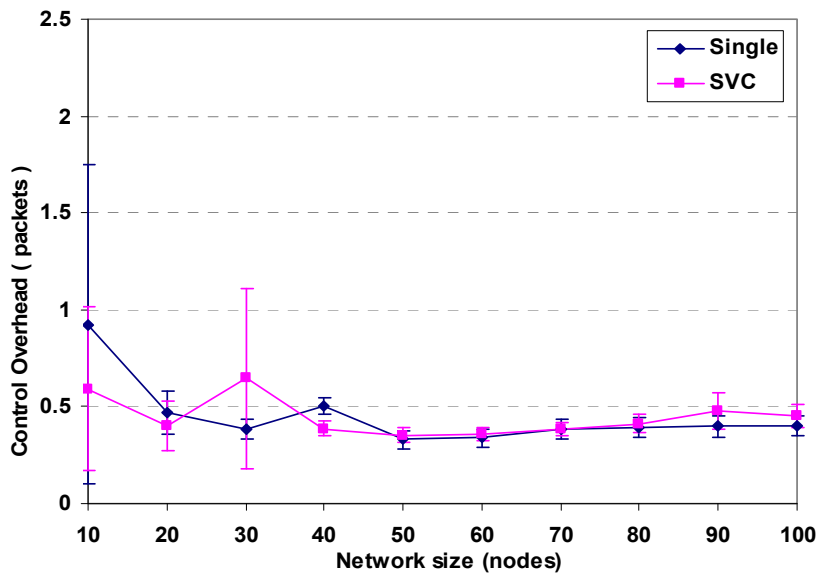

Figure 7. Varying network size with average overhead for single and layered video transport, showing $95 \%$ confidence intervals.

The packet overhead is a measure of the efficiency of ad hoc routing. Packet overhead in Fig. 7 is the mean number of control packets per packet. Fig. 7 shows a familiar story in which overhead is very low and stable for medium to high node densities but is erratic at lower densities. Equally in Fig. 8, higher speeds result in erratic efficiency levels. From inspection of the results, the large error bars were found to be the result of bi-modal overhead levels.

Table 2 summarizes findings published in [27], correlating packet loss ratio with video streaming Quality-of-Experience (QoE) in mobile ad hoc networks. We note that QoE is sensitive to packet loss and that $14 \%$ is the threshold applied. However, QoE is somewhat different from video quality as it takes into account screen size, audio quality and other attendant factors in streaming. In Table 3, the percentage packet loss ratios are given for a single sample set of simulations with single-layer delivery of video and in Table 4 for the same simulation the packet loss ratios are shown for both streams for layered video delivery. In Table 4, the columns marked ' $\mathrm{B}$ ' are packet loss ratios for the base layer stream only, while the columns marked ' $\mathrm{B}+\mathrm{E}$ ' are the figures for the combined stream of base and enhancement layer packets. The

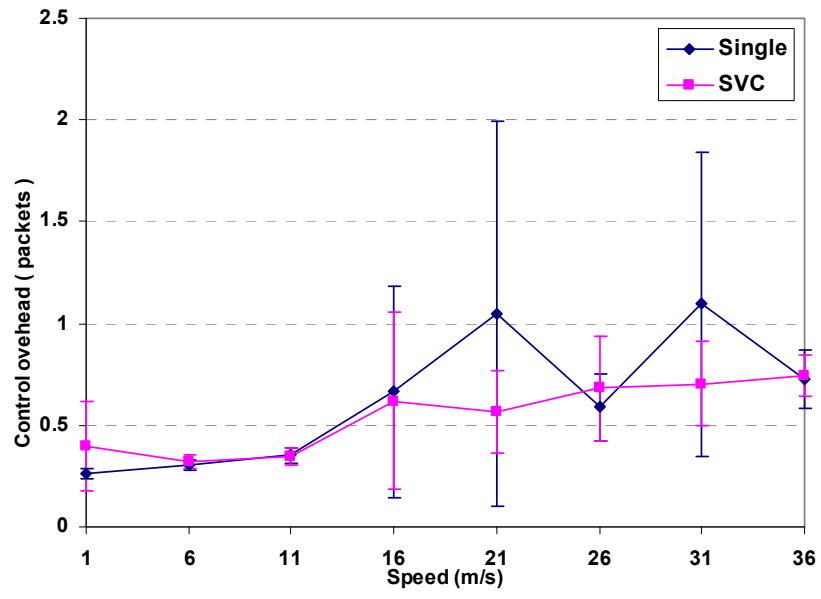

Figure 8. Varying node speed with average overhead for single and layered video transport, showing $95 \%$ conf. intervals.

Table 2. QoE acceptability levels [27].

\begin{tabular}{|c|c|c|}
\hline $\begin{array}{c}\text { Packet loss } \\
\text { ratio (\%) }\end{array}$ & $\begin{array}{c}\text { QoE } \\
\text { acceptability (\%) }\end{array}$ & Video quality playback \\
\hline 0 & 84 & Smooth \\
14 & 61 & Brief interruptions \\
18 & 41 & Frequent interruptions \\
25 & 31 & Intolerable interruptions \\
31 & 0 & Stream breaks \\
\hline
\end{tabular}

sample selected has no special qualities and, in fact, as far as we could ascertain it was typical. From the Tables it is apparent that in this example some destination nodes such as $\mathrm{C}$ and $\mathrm{E}$ happen to suffer from packet loss particularly badly. This is attributed to their positions during some of the simulation set. In Table 4, column ' $R$ ' represents the packet loss ratio from the combined recovered stream. That is if a base layer packet was missing in one stream it was substituted for from the other stream. Comparing across the Tables, by inspection on a case by case basis, we found that there were occasions in which the recovered stream was adequate for reconstruction while the single layer was not. These occasions are more numerous than when the singlelayer packet loss ratio is lower than the recovered stream, when one takes into account that above $14 \%$ from Table 2, the QoE is likely to be unsatisfactory anyway whatever the packet loss ratio. Furthermore, this analysis does not distinguish between loss of enhancement and base layer packets in Table 4 column ' $R$ ' and if it had then the advantage to the layered streaming would be greater. In summary, detailed analysis of packet loss ratios confirms the summary network statistics.

\section{CONCLUSIONS}

In this paper, layered video over multiple paths was shown to be an efficient way to transport video clips over an ad hoc network. We have proposed layered coding without error control by simply duplicating base layer distribution in each of the streams. Though simple, comparison with single-layer and single path distribution points to a consistent reduction in packet loss and commensurate levels of end-to-end delay and control packet overhead. Peer-topeer streaming, as simulated by us, appears to be a natural way of multicasting video within these networks, as it too relies on multi- 
path distribution. Moreover, as multicast video distribution is newly introduced into cellular networks, then infrastructure-less distribution will complement this service. There may also be other roles for ad hoc video distribution: across emergency networks and in vehicular networks. ARQ is not the only form of protection that can be applied to a base layer and in future work alternatives such as Forward Error Correction and possibly adaptive modulation will be compared with our method. Though we have assessed our results based on network statistics, the loss pattern will also have an effect on video quality and we also intend to take this into account in future work.

\section{REFERENCES}

[1] Wu, H., Qiao, C., De, S. and Tonguz, O. 2001. Integrated cellular and ad hoc relaying service: iCAR. IEEE J. on Sel. Areas in Comms. 19(10), 2105-2113.

[2] Lu, W.-F. and Wu, M. 2002. MADF: Mobile-Assisted data forwarding for wireless data networks, J. of Comms. and Networks, 6(3), 216-233.

[3] Afzal. J., Stockhammer, T., Gasiba, T., Xu, W. 2006. Video Streaming over MBMS: A System Design Approach. J. of Multimedia, 1(5), 25-35.

[4] Oliveira, L. B., Siqueira, I. and Loureiro, A. A. F. 2005. On the performance of ad hoc routing protocols under a peer-topeer application. J. of Parallel and Distributed Computing, 65(11), 1337 - 1347.

[5] Yan, L. 2005. Can P2P benefit from MANET? Performance evaluation from users' perspective. In Proceedings of Int. Conf. on Mobile Sensor Networks, 1026-1035.

[6] Chen, J., Chan, S. H. and Li, V. O. K. 2004. Multipath routing for video delivery over bandwidth-limited networks. IEEE J. on Selected Areas in Comms., 22(10), 1920-1932.

[7] Reibman, A. R. Jafarkhani, H., Orchard, M. Y. and Wang, Y. 2001. Multiple description video using rate-distortion splitting. In proceedings of IEEE Int. Conf. on Image Processing, 971-981.

[8] Jurca, D., Chakareski, J., Wagner, J. and Frossard, P. 2007. Enabling Adaptive Video Streaming in P2P Systems. IEEE Comms. Mag., 45(6), 108-114.

[9] Wang, Y., A. R. Reibman, A. R.and Lee, S. 2005. Multiple description coding for video delivery, Proceedings of the IEEE, 93(1), 57-70.

[10] Apostolopoulos, J. 2001. Reliable video communication over lossy packet networks using multiple state encoding and path diversity. Visual Comms.: Image Processing, 392-409.

[11] Ghanbari, M. 2003. Standard codecs: Image compression to advanced codecs. IET Press, Stevenage, UK.

[12] Mao, S., Lin, S. S. S. Panwar, S. S. and Y. Wang, Y. 2001. Reliable transmission of video over ad-hoc networks using automatic repeat request and multi-path transport. In Proceedings of IEEE Vehicular Technology Conf., 615-619.

[13] Mao, S., Lin, S. Panwar, S. S., Wang, Y. and Celebi, E. 2003. Video transport over ad hoc networks: multistream coding with multipath transport. IEEE J. on Selected Areas in Comms., 21(4), 1721-1737.

[14] Setton, E., Zhu, X. and Girod, B. 2004. Congestionoptimized multipath streaming of video over ad hoc wireless network. In Proceedings of IEEE Int. Conf. on Multimedia and Expo, 1619-1622.

[15] Wei, W. and Zakhor, A. 2004. Multipath unicast and multicast video communication over wireless ad hoc networks. In Proceedings of Int. Conf. on Broadband Networks, 496-505.

[16] Broch, J., Maltz, D. A., Johnson, D. B. et al. 1998. A performance comparison of multi-hop wireless ad hoc network routing protocols. In Proceedings of ACM Mobicom, 85-97

[17] Wei, W. and Zakhor, A. 2006. Path selection for multi-path streaming in wireless ad hoc networks. In Proceedings of Int Conf. on Image Processing, 3045-3048.

[18] Wei, W. and Zakhor, A. 2009. Interference aware multipath selection for video streaming in wireless ad hoc networks. IEEE Trans. on Circ. and Syst. for Video Technology, 19(2), 165-178.

[19] Chakereseki, J., Han, S. and Girod, B. 2005. Layered coding vs. multiple descriptions for video streaming over multiple paths. Multimedia Systems, online journal.

[20] Schierl, T., Jhansen, S.; Perkis, A. and Wiegand, T. 2008. Rateless scalable video coding for overlay multisource streaming in MANETs, J. Vis. Commun. Image R., 19, 500507

[21] Liu, Y., Guo, Y. and Liang, C. 2008. A survey on peer-topeer video streaming systems. J. of P2P Networking and Applications, 1(1), 18-28.

[22] Zeng, X., Bagrodia, R. and Gerla, M. 1998. GloMoSim: A library for parallel simulation of large-scale wireless networks. In Proceedings of $12^{\text {th }}$ Workshop on Parallel and Distributed Simulations. 154-161.

[23] Perkins, C. E. and Royer, E. M. 1999. Ad hoc on-demand distance vector routing (AODV). In Proceedings of $2^{\text {nd }}$ IEEE Workshop on Mobile Computing Systems and Applications. 90-100.

[24] Lee, S. J. and Gerla, M. 2001. Split multipath routing with maximally disjoint paths in ad hoc networks. In Proceedings of Int. Conf. on Communications. 3201-320

[25] Karlsson, J., Li, H. and Erikson, J. 2005. Real-time video over wireless ad-hoc networks. In Proceedings of $14^{\text {th }}$ Int. Conf. on Computer Comms. and Networks, 596-607.

[26] Agboma, F. and Liotta, A. (2008) Quality of Experience for mobile TV services. Ahmad, A. H. A. and Ibrahim, I. K. (eds.) Multimedia Transcoding for Mobile and Wireless Networks, Info. Science Reference, Hershey, PA, 178-197.

[27] Agboma, F., Smy, M. and Liotta, A. (2008) QoE analysis of a peer-to-peer television system. In Proceedings of IADIS Int. Conf. on Telecommunications, Networks and System 
Table 3. Packet loss ratio for single-layer coding for a single sample simulation.

\begin{tabular}{|c|l|l|l|l|l|}
\hline & \multicolumn{5}{|c|}{ Packet loss ratio (\%) at receivers-Single layer coding } \\
\hline Node & Peer C & Peer D & Peer E & \multicolumn{1}{c|}{ Peer F } & Peer G \\
\hline $\mathbf{1 0}$ & 100 & 2 & 100 & 100 & 1 \\
\hline $\mathbf{2 0}$ & 4 & 0 & 2 & 56 & 2 \\
\hline $\mathbf{3 0}$ & 0 & 0 & 1 & 1 & 2 \\
\hline $\mathbf{4 0}$ & 100 & 0 & 90 & 1 & 1 \\
\hline $\mathbf{5 0}$ & 100 & 0 & 93 & 0 & 0 \\
\hline $\mathbf{6 0}$ & 100 & 0 & 99 & 0 & 0 \\
\hline $\mathbf{7 0}$ & 30 & 0 & 95 & 0 & 0 \\
\hline $\mathbf{8 0}$ & 26 & 0 & 94 & 0 & 0 \\
\hline $\mathbf{9 0}$ & 100 & 0 & 91 & 0 & 0 \\
\hline $\mathbf{1 0 0}$ & 100 & 0 & 90 & 0 & 0 \\
\hline Speed & & & & & \\
\hline $\mathbf{1}$ & 100 & 0 & 100 & 0 & 0 \\
\hline $\mathbf{6}$ & 100 & 0 & 99 & 0 & 0 \\
\hline $\mathbf{1 1}$ & 100 & 0 & 94 & 1 & 0 \\
\hline $\mathbf{1 6}$ & 2 & 0 & 0 & 0 & 0 \\
\hline $\mathbf{2 1}$ & 5 & 0 & 2 & 0 & 0 \\
\hline $\mathbf{2 6}$ & 0 & 0 & 4 & 4 & 0 \\
\hline $\mathbf{3 1}$ & 0 & 0 & 7 & 2 & 0 \\
\hline $\mathbf{3 6}$ & 3 & 0 & & & 0 \\
\hline
\end{tabular}

Table 4. Packet loss ratios for SVC for a single sample simulation.

\begin{tabular}{|c|c|c|c|c|c|c|c|c|c|c|c|c|c|c|c|}
\hline \multicolumn{16}{|c|}{ Packet Loss ratio (\%) at each peer-Layered coding } \\
\hline Node & \multicolumn{3}{|c|}{ Peer C } & \multicolumn{3}{|c|}{ Peer D } & \multicolumn{3}{|c|}{ Peer E } & \multicolumn{3}{|c|}{ Peer F } & \multicolumn{3}{|c|}{ Peer G } \\
\hline & $\mathbf{B}$ & $\mathbf{B}+\mathbf{E}$ & $\mathbf{R}$ & $\mathbf{B}$ & $\mathbf{B}+\mathbf{E}$ & $\mathbf{R}$ & $\mathbf{B}$ & $\mathbf{B}+\mathbf{E}$ & $\mathbf{R}$ & B & $\mathbf{B}+\mathbf{E}$ & $\mathbf{R}$ & B & $\mathbf{B}+\mathbf{E}$ & $\mathbf{R}$ \\
\hline 10 & 100 & 100 & 100 & 0 & 93 & 52 & 0 & 93 & 52 & 100 & 67 & 67 & 0 & 40 & 19 \\
\hline 20 & 0 & 29 & 17 & 19 & 40 & 28 & 21 & 7 & 5 & 48 & 78 & 78 & 58 & 3 & 2 \\
\hline 30 & 8 & 1 & 1 & 4 & 22 & 16 & 0 & 3 & 2 & 0 & 0 & O & 0 & 1 & 0 \\
\hline 40 & 0 & 0 & 0 & 0 & 0 & 0 & 0 & 3 & 2 & 0 & 0 & 0 & 0 & 0 & 0 \\
\hline 50 & 100 & 0 & 0 & 0 & 0 & 0 & 0 & 1 & 1 & 0 & 0 & O & 0 & 0 & $\mathbf{0}$ \\
\hline 60 & 100 & 0 & 0 & 0 & 0 & 0 & 0 & 1 & 1 & 0 & 0 & 0 & 0 & 0 & 0 \\
\hline 70 & 97 & 42 & 42 & 3 & 1 & 1 & 5 & 1 & 1 & 0 & 0 & 0 & 0 & 0 & 0 \\
\hline 80 & 97 & 42 & 42 & 3 & 1 & 1 & 4 & 2 & 2 & 0 & 0 & 0 & 0 & 0 & 0 \\
\hline 90 & 100 & 93 & 93 & 0 & 1 & 1 & 1 & 0 & 0 & 0 & 0 & 0 & 0 & 0 & 0 \\
\hline 100 & 100 & 93 & 93 & 0 & 1 & 1 & 1 & 0 & 0 & 0 & 0 & 0 & 0 & 0 & 0 \\
\hline \multicolumn{16}{|l|}{ Speed } \\
\hline 1 & 100 & 93 & 93 & 1 & 0 & 0 & 0 & 1 & 1 & 0 & 0 & 0 & 0 & 0 & $\mathbf{0}$ \\
\hline 6 & 100 & 0 & 0 & 4 & 1 & 1 & 1 & 62 & 40 & 0 & 0 & O & 0 & 0 & 0 \\
\hline 11 & 1 & 0 & 0 & 1 & 1 & 1 & 1 & 0 & 0 & 0 & 0 & 0 & 0 & 0 & 0 \\
\hline 16 & 0 & 1 & 0 & 5 & 3 & 2 & 1 & 1 & 1 & 0 & 0 & 0 & 0 & 0 & 0 \\
\hline 21 & 3 & 0 & $\mathbf{0}$ & 5 & 2 & 1 & 10 & 3 & 2 & 0 & 0 & 0 & 0 & 0 & 0 \\
\hline 26 & 8 & 1 & 1 & 8 & 16 & 10 & 37 & 12 & 9 & 0 & 0 & 0 & 0 & 0 & 0 \\
\hline 31 & 4 & 95 & 57 & 0 & 6 & 2 & 5 & 4 & 3 & 0 & 1 & 1 & 0 & 0 & 0 \\
\hline 36 & 12 & 4 & 3 & 8 & 28 & 20 & 11 & 15 & 11 & 0 & 12 & 6 & 0 & 0 & 0 \\
\hline
\end{tabular}

